\section{Networking on the fly}

There are now many ways in which genes that are mutated in particular cancer types can be identified, including starting from less genetically complex organisms such as Drosophila melanogaster. Kevin White and colleagues have taken this approach and have identified SPOP as a protein that is overexpressed in renal cell carcinoma (RCC).

D. melanogaster has proved particularly useful for identifying gene networks that are conserved throughout evolution. White and colleagues started with two pairrule genes - eve and $\mathrm{ftz}$ - homeobox genes that are part of a gene network often disrupted in human disease. Using a variety of information about Eve and Ftz, they built a predictive gene network model. Initially, gene expression patterns from wild-type embryos or embryos with mutated eve or $f t z$ taken over a time course of 2-7 hours after egg laying (AEL) were compared. Chromatin immunoprecipitation analysed on DNA microarrays (ChIP on chip) was also used to map Eve and Ftz DNA binding sites 2 hours AEL. Genes that were both differentially expressed and identified by the ChIP-chip approach were considered as putative targets: 137 Ftz target genes and 98 Eve target genes. To extend the network further, the authors then added in information on the target genes obtained from automated literaturemining techniques and yeast twohybrid protein-protein interaction data. The resulting network contained 4,084 genes and proteins and 6,648 interactions between them.

Having tested that specific links within the network behave as expected, the authors analysed the 150 genes that have validated human homologues. The top apoptosis. candidate (a major network hub) was roadkill $(r d x)$, which is $79 \%$ identical to the human protein SPOP. The network model indicated that D-SPOP $(\mathrm{Rdx})$ is a Ftz target 2-3 hours AEL and then becomes an Eve target 6-7 hours AEL, and that D-SPOP interacts with the Jun kinase phosphatase Puckered (Puc). Further analyses indicated that D-SPOP and Puc interact and this is important for the regulation of Eiger (tumour necrosis factor)induced apoptosis in neurons. Indeed, the authors found that D-SPOP, like human SPOP, can induce protein ubiquitylation and degradation, and D-SPOP induced the degradation of Puc, which functions to inhibit Eiger-mediated JUN N-terminal kinase-induced

As homologues of Eve and Ftz targets are involved in tumorigenesis in humans, the authors investigated whether SPOP shows altered expression in tumours using a human tissue microarray. They found that SPOP

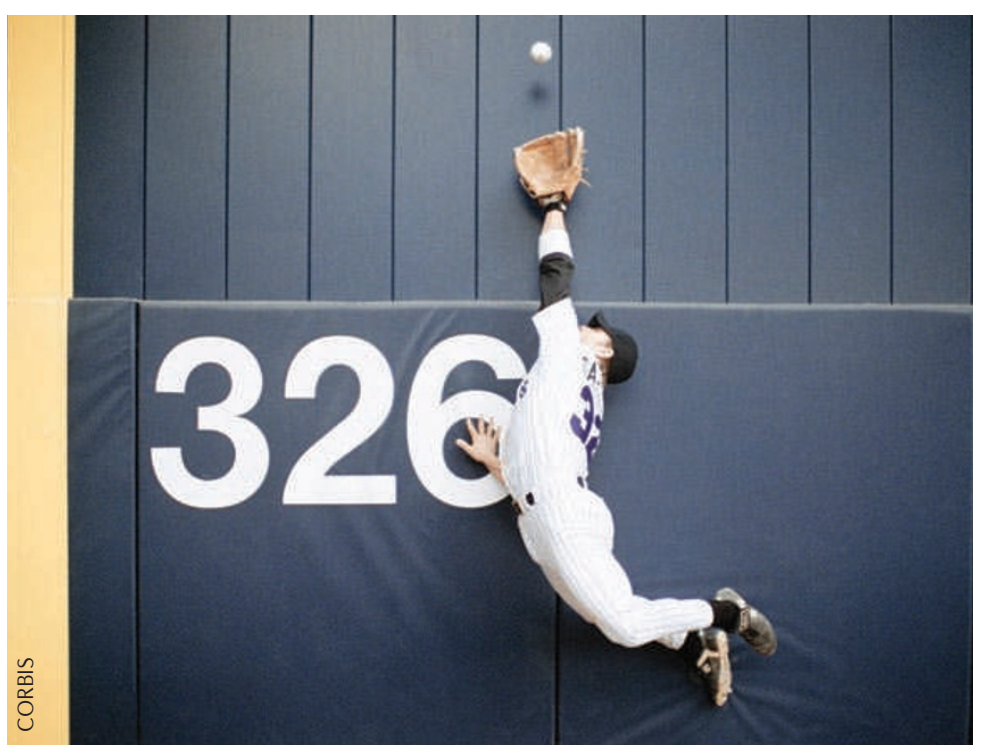

is highly expressed in $85 \%$ of RCCs, whereas the protein is expressed at a low level in normal kidney tissue. Moreover, they found that SPOP expression can be used to identify different types of RCC. In clear cell RCC, which in some cases can be difficult to distinguish from other types of RCC, $99 \%$ of cases were positive for SPOP expression, as were $86 \%$ of chromophobe RCCs, whereas only $22 \%$ of papillary-type RCCs were positive.

The authors conclude that analysing gene networks on the basis of information in D. melanogaster is an effective method for understanding the biological function of these networks, for identifying conserved gene networks and identifying new genes within these networks that are deregulated in human disease.

Nicola McCarthy

ORIGINAL RESEARCH PAPER Liu, J. et al. Analysis of Drosophila segmentation network identifes a JNK pathway factor overexpressed in kidney cancer. Science 22 Jan 2009 (doi 10.1126/ science1157669) 